doi.org/ I0.51891/rease.v7iro.2692

\title{
A IMPORTÂNCIA DO REVESTIMENTO ENTÉRICO EM CÁPSULAS
}

\author{
Caroline Nunes Claro ${ }^{1}$ \\ Alex Sandro Rodrigues Baiense ${ }^{2}$
}

RESUMO: O objetivo do trabalho foi analisar através de revisão bibliográfica a importância do revestimento entérico em cápsulas. $O$ revestimento entérico é valioso para colocar uma barreira sobre um medicamento para evitar que ele se dissolva até passar pelo estômago. A palavra entérica significa relacionar-se ou estar dentro do intestino. Com um revestimento entérico, o medicamento é liberado dependendo do valor do $\mathrm{pH}$ no trato gastrointestinal que inicia a dissolução do acabamento específico aplicado. A estratégia de usar o $\mathrm{pH}$ como gatilho para a liberação específica de um produto surge do aprendizado da variação do $\mathrm{pH}$ ao longo do trato gastrointestinal. Alguns polímeros são utilizados para o revestimento de filmes de liberação entedia tal como o Acetado de Ftalato de celulose, apresentando uma rápida dissolução em $\mathrm{pH}>6$, Hidroxil propil metil celulose ftalato que possui sabor imperceptível é solúvel em meio aquoso, com $\mathrm{pH}$ de 5,0 - 5,5, Succinato de acetato de hidroxil propil metil celulose sendo insolúvel em meio ácido, mas solúvel em $\mathrm{pH}$ neutro, Acetato ftalato insolúvel em fluidos gástricos, mas incha e rapidamente solúvel na parte superior do intestino e por fim o ácido Metacrílico que permitem o direcionamento de áreas específicas do intestino. O tipo do estudo é uma revisão bibliográfica, pesquisas do tipo tem o objetivo primordial à exposição dos atributos de determinado fenômeno ou afirmação entre suas variáveis. Assim, recomenda-se que apresente características do tipo: analisar a atmosfera como fonte direta dos dados e o pesquisador como um instrumento interruptor; não agenciar o uso de artifícios e métodos estatísticos, tendo como apreensão maior a interpretação de fenômenos e a imputação de resultados, o método deve ser o foco principal para a abordagem e não o resultado ou o fruto, a apreciação dos dados deve ser atingida de forma intuitiva e indutivamente através do pesquisador.

Palavras-chave: Revestimento entérico. Farmácia. Cápsulas.

ABSTRACT: The aim of this work was to analyze, through a literature review, the importance of enteric coating in capsules. The enteric coating is valuable for putting a barrier over a drug to keep it from dissolving through the stomach. The word enteric means to relate to or be inside the intestine. With an enteric coating, the drug is released depending on the $\mathrm{pH}$ value in the gastrointestinal tract which initiates the breakdown of the specific applied finish. The strategy of using $\mathrm{pH}$ as a trigger for the specific release of

\footnotetext{
IAluna do curso Bacharel em Farmácia da Universidade Iguaçu. E-mail: c.ccla@hotmail.com

${ }^{2}$ Professor, farmacêutico industrial CRFRJ 7275 E-mail: alexbaiense@gmail.com
} 
a product arises from learning how to change $\mathrm{pH}$ along the gastrointestinal tract. Some polymers are used to coat bored release films such as cellulosephthalate Acete, presenting a rapid dissolution at $\mathrm{pH}>6$, Hydroxyl propyl methyl cellulose phthalate that has imperceptible flavor is soluble in aqueous medium, with $\mathrm{pH}$ of 5.0 - 5.5, Hydroxyl acetate succinate propyl methyl cellulose being insoluble in acid medium, but soluble at neutral $\mathrm{pH}$, Phthalate acetate insoluble in gastric fluids, but swells and quickly soluble in the upper part of the intestine and finally Metacrylic acid that allow the direction of specific areas of the intestine. The type of study is a bibliographic review, research of this type has the primary objective of exposing the attributes of a given phenomenon or statement among its variables. Thus, it is recommended that it presents characteristics such as: analyzing the atmosphere as a direct source of data and the researcher as a switch instrument; not to broker the use of statistical artifices and methods, having as a greater apprehension the interpretation of phenomena and the imputation of results, the method should be the main focus for the approach and not the result or the fruit, the appreciation of the data should be achieved from intuitively and inductively through the researcher.

Keywords: Enteric coating. Pharmacy. Capsules.

\section{INTRODUÇÃO}

Avanços científicos levam à criação de medicamentos, que são ferramentas valiosas para os médicos no atendimento ao paciente. Os medicamentos podem curar as pessoas, evitar doenças e aliviar a dor, apenas para citar algumas coisas, mas podem vir em formulações que não são amigáveis para o trato gastrointestinal (GI). Outras vezes, os medicamentos precisam de proteção contra o ambiente severo do estômago, que pode danificá-los ou destruí-los antes que tenham a chance de fazer efeito. Além disso, algumas drogas são instáveis se deixadas em seu estado inalterado. Superar esses obstáculos significa que um processamento técnico complexo é necessário antes que os medicamentos apareçam nas prateleiras das farmácias (ARAI, GALLERANI, 20II).

O desafio para os fabricantes de medicamentos é transformar um produto químico ou natural valioso em um medicamento eficaz e seguro para o consumo do paciente. Os medicamentos devem chegar aonde precisam para fazer seu trabalho, sem prejudicar outras áreas do corpo. Comprimidos, cápsulas, líquidos, pós, suspensões, injeções e enemas são alguns dos principais modos de aplicação dos medicamentos em nossos corpos para que possam fazer seu trabalho. A variação dos tipos de revestimento colocados nos comprimidos e cápsulas é uma forma de controlar a medicação dentro do corpo (DEPONTI, 2012). 
Os revestimentos de medicamentos servem a muitos propósitos; eles fornecem cor para estabelecer a identidade da marca, mascaram o sabor amargo, fornecem uma capa dura para compostos quebradiços ou quebradiços para que o medicamento não se dissolva na boca e aumentam a vida útil do produto. Os revestimentos variam em consistência e formulação para permitir a liberação imediata, retardada ou sustentada e a liberação direcionada (MENDONÇA, 2010).

Os acabamentos podem consistir em misturas de polímeros, plastificantes, pigmentos, opacificantes, deslizantes, aglutinantes, agentes antiaderentes, mecanismos antiespumantes, surfactantes, enchimentos e extensores. Vários produtos químicos que precisam de ajuda para passar pelo estômago incluem aqueles que são sensíveis à umidade, degradam-se em diferentes temperaturas, param de funcionar com o tempo, são especificamente vulneráveis aos sucos gástricos ou podem causar danos ao estômago ou outras partes do trato digestivo (MIRANDA, 2015).

O revestimento entérico é valioso para colocar uma barreira sobre um medicamento para evitar que ele se dissolva até passar pelo estômago. A palavra entérica significa relacionar-se ou estar dentro do intestino. Com um revestimento entérico, o medicamento é liberado dependendo do valor do $\mathrm{pH}$ no trato gastrointestinal que inicia a quebra do acabamento específico aplicado. A estratégia de usar o $\mathrm{pH}$ como gatilho para a liberação específica de um produto surge do aprendizado da variação do $\mathrm{pH}$ ao longo do trato gastrointestinal (TESTA, 2013).

Produtos naturais como óleo de peixe e óleo de hortelã-pimenta são benéficos para o consumidor quando um revestimento entérico permite a liberação no intestino, mas, quando consumidos sem revestimento, esses produtos seriam liberados no estômago, possivelmente diminuindo a eficácia e causando efeitos indesejados no trato gastrointestinal superior. Alguns compostos de medicamentos podem conter uma camada de invólucro que libera ingredientes ativos imediatamente no estômago, com um revestimento entérico interno, que retarda a liberação do núcleo interno, geralmente um medicamento diferente, até mais abaixo no trato gastrointestinal.

O objetivo do trabalho foi analisar através de revisão bibliográfica a importância do revestimento entérico em cápsulas.

O tipo do estudo é uma revisão bibliográfica, pesquisas do tipo tem o objetivo primordial à exposição dos atributos de determinado fenômeno ou afirmação entre suas 
variáveis. Assim, recomenda-se que apresente características do tipo: analisar a atmosfera como fonte direta dos dados e o pesquisador como um instrumento interruptor; não agenciar o uso de artifícios e métodos estatísticos, tendo como apreensão maior a interpretação de fenômenos e a imputação de resultados, o método deve ser o foco principal para a abordagem e não o resultado ou o fruto, a apreciação dos dados deve ser atingida de forma intuitiva e indutivamente através do pesquisador.

\section{REVESTIMENTO ENTÉRICO}

O revestimento entérico é um polímero aplicado à medicação oral. Ele serve como uma barreira para evitar que os ácidos gástricos no estômago dissolvam ou degradem os medicamentos após sua ingestão. Sem proteção entérica completa, muitos medicamentos se desintegrariam rapidamente nos ácidos estomacais. $\mathrm{O}$ efeito pretendido seria reduzido ou totalmente eliminado. Medicamentos diferentes atuam de maneiras diferentes, mas vários tipos de medicamentos precisam chegar intactos ao trato intestinal antes de liberar seus ingredientes (TESTA, 2013).

O revestimento cria uma liberação retardada para várias formas de 1674 dosagem. Graças a esse processo, as empresas farmacêuticas podem garantir que os medicamentos cheguem ao estômago e ao intestino delgado ou a outros estágios da digestão que precisam ser alcançados. Por meio da combinação de proteção entérica e ingredientes de liberação rápida, os profissionais médicos agora podem determinar com precisão quando um medicamento entra em ação (MIRANDA, 2015).

Este revestimento faz mais do que proteger as drogas, no entanto. Ele também defende o estômago e é o revestimento contra os danos que certos medicamentos podem causar. A classe de analgésicos conhecidos como AINEs está entre os mais comumente usados no mercado hoje (MENDONÇA, 2010).

Qualquer pessoa que tome AINEs regularmente para o alívio da dor ou de condições como artrite corre o risco de desenvolver úlceras estomacais devido à irritação que os medicamentos podem criar. O American College of Gastroenterology declarou que até $25 \%$ das pessoas que tomam AINEs regularmente desenvolverão úlceras, sangramento no estômago ou lesões no trato gastrointestinal superior do trato gastrointestinal. Essas lesões contribuem para mais de Ioo.ooo visitas ao hospital todos os anos (MENDONÇA, 2010). 
A indústria farmacêutica abordou esse risco desenvolvendo uma nova tecnologia de entrega de medicamentos e revestindo os comprimidos. Os medicamentos revestidos para a dor atuam na prevenção ou redução da irritação que causa esses ferimentos internos. As cápsulas ou comprimidos com revestimento entérico impedem a ativação dos ingredientes do medicamento até que estejam com segurança além do estômago (TESTA, 2013).

Algumas empresas estão até aplicando revestimentos entéricos a suplementos, como comprimidos de óleo de peixe ou cápsulas de ácido graxo ômega-3. Nesta aplicação, o revestimento ainda impede que a cápsula seja digerida no estômago. No entanto, desta vez, o revestimento é usado para evitar o refluxo ou arroto de peixe que geralmente ocorre após a ingestão de comprimidos de óleo de peixe (MIRANDA, 2015).

Um revestimento entérico, também conhecido como revestimento gastroresistente, é uma barreira aplicada ao medicamento oral que controla o local no trato digestivo onde é absorvido. O termo "entérico" refere-se ao intestino delgado; portanto, os revestimentos entéricos resistem à quebra da medicação antes que ela atinja o intestino delgado. Os revestimentos entéricos são utilizados quando o fármaco é inativado ou destruído na secreção ácida do estômago ou é particularmente irritante para a mucosa gástrica ou quando o desvio do estômago aumenta substancialmente a absorção do fármaco (MIRANDA, 2015).

As primeiras abordagens para a preparação de formas de dosagem entérica envolviam o tratamento de cápsulas de gelatina com formalina ou o revestimento de comprimidos com goma-laca. Ambas as abordagens não eram confiáveis, uma vez que a solubilidade da membrana (que é responsável pelo efeito entérico) pode ser imprevisível (TESTA, 2013).

\section{I.I RAZÕES PARA A APLICAÇÃO DE REVESTIMENTOS ENTÉRICOS}

Os revestimentos entéricos são aplicados às formas de dosagem pelas seguintes razões:

i. Para proteger as substâncias medicamentosas lábeis em ácido do $\mathrm{pH}$ do ácido gástrico. Essas substâncias medicamentosas incluem eritromicina, pancreatina, omeprazol etc.; 
ii. Para prevenir desconforto / ulceração gástrica ou náusea devido à irritação causada por certos medicamentos, como aspirina e certos compostos antiinflamatórios não esteróides;

iii. Para entregar medicamentos que são absorvidos de forma ideal no intestino delgado para seu local de absorção primário em sua forma mais concentrada;

iv. Para fornecer um componente de liberação retardada para comprimidos de ação repetida (TESTA, 2013).

Alguns dos principais atributos dos materiais de revestimento entérico incluem:

Resistência aos fluidos gástricos; Pronta suscetibilidade ou permeabilidade aos fluidos intestinais; Alta compatibilidade com outros aditivos de revestimento de filme e o comprimido sendo revestido; Capacidade de formar filme contínuo com propriedades mecânicas adequadas; Facilidade de aplicação sem equipamento especial; Capacidade de ser impresso ou permitir que o filme seja aplicado a comprimidos gravados; Atóxico sem atividade farmacológica e Estável sozinho e em solução de revestimento (MIRANDA, 2015).

\section{I.I. POLÍMEROS USADOS PARA REVESTIMENTO DE FILME DE} LIBERAÇÃO ENTÉRICA

\section{I.I.I. Acetato Ftalato De Celulose (CAP)}

Acetato ftalato de celulose, também conhecido como celacefato, é o polímero de revestimento entérico sintético mais antigo e mais amplamente utilizado. Foi patenteado como agente entérico em 1940 pela Eastman Kodak Company. O CAP é sintetizado pela reação de um éster de acetato parcial de celulose com anidrido ftálico na presença de uma base orgânica terciária, como a piridina, ou um ácido forte, como o ácido sulfúrico (TESTA, 2013). 


\section{Figura r: ESTRUTURA QUÍMICA ANIDRIDO FTÁLICO}

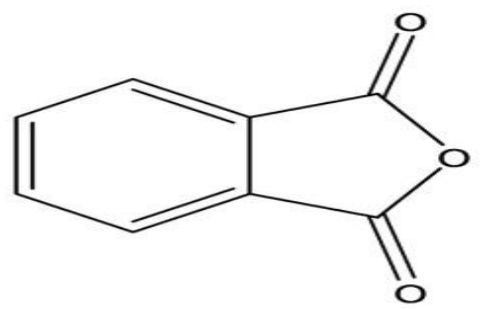

No polímero resultante, dos grupos hidroxila livres contribuídos por cada unidade de glicose da cadeia de celulose, aproximadamente metade são acilados e um quarto esterificados com um dos dois grupos de ácido carboxílico da porção ftalato. $\mathrm{O}$ segundo grupo de ácido carboxílico sendo livre para formar sais e, portanto, serve como base para seu caráter entérico (ARAI, GALLERANI, 20II). Como o grau de substituição pode levar a alterações nas propriedades do CAP, as especificações para a composição do CAP foram estabelecidas para garantir um desempenho mais uniforme de lote para lote. De acordo com as especificações da Farmacopeia do Estado dos Estados Unidos (USP), o CAP deve conter $21,5-26,0 \% \mathrm{p} / \mathrm{p}$ de conteúdo de acetil e 30,0 - 36,0\% p / p de grupos ftalil na estrutura da celulose, conforme calculado em uma base anidra (MIRANDA, 2015).

CAP é insolúvel em água, hidrocarbonetos e hidrocarbonetos clorados, mas solúvel em cetonas, éteres, álcoois, ésteres e em certas misturas de solventes. Apresenta rápida dissolução em $\mathrm{pH}>6$ e é relativamente permeável à umidade e sucos gástricos. Devido à sua alta permeabilidade à umidade, o CAP é suscetível à decomposição hidrolítica (TESTA, 2013).

As moléculas de ácido ftálico e acético podem hidrolisar durante o armazenamento e comprometer significativamente o grau de proteção entérica que o revestimento de filme fornece. A adição de um agente plastificante demonstrou melhorar a resistência à água dos filmes CAP (ARAI, GALLERANI, 2oII).

CAP é compatível com a maioria dos plastificantes solúveis em água e insolúveis com dietil ftalato (DEP), tributil citrato (TBC), trietil citrato (TEC), tributirina e triacetina sendo os mais comumente usados; tipicamente na faixa de $25-35 \%$ em peso de polímero seco. Como outros ftalatos, o CAP é suscetível à hidrólise sob alta temperatura e umidade. CAP está comercialmente disponível como um pó branco da Eastman Chemical Co. Uma versão de dispersão de látex com 30\% de sólidos de CAP (Aquacoat ${ }^{\circledR} \mathrm{CPD}$ ) também está disponível para 
revestimento entérico aquoso de comprimidos, grânulos, cápsulas de gelatina dura e mole (TESTA, 2013).

I.I.2. Trimelitato de acetato de celulose (CAT)

Quimicamente, este polímero apresenta uma forte semelhança com o CAP. É formado pelo mesmo processo de síntese do CAP com anidrido trimelítico como grupo substituinte no lugar do anidrido ftálico. Os valores típicos para a substituição de timelitil e acetil são 29,0\% e 22,4\%, respectivamente (ARAI, GALLERANI, 20II).O anidrido trimelítico contém um grupo carboxila livre adicional sobre o anidrido ftálico e, portanto, CAT contém uma concentração maior de grupos ácidos para um determinado grau de substituição do que CAP, tornando-o mais solúvel em meio aquoso. Além disso, o pKa de CAT está entre 4, I e 4,3, o que é ligeiramente inferior ao CAP. Com um valor de pKa relativamente baixo e maior concentração de grupo funcional (MIRANDA, 2015)

Figura 2: ESTRUTURA QUÍMICA ANIDRIDO TRIMELÍTICO

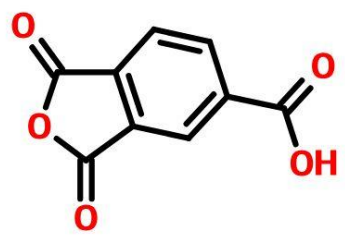

CAT é o derivado de celulose entérica mais solúvel, com o início da dissolução ocorrendo em $\mathrm{pH}$ 4,7-5,0. Esta propriedade útil torna o CAT ideal para a liberação direcionada do fármaco para as regiões proximais do intestino delgado. CAT está disponível comercialmente como um pó branco da Eastman Chemical Co. Para obter os melhores resultados de revestimento entérico do processamento aquoso, são recomendadas soluções amoniacais de CAT em água. As considerações do plastificante para CAT são idênticas às do CAP (TESTA, 2013). 


\subsubsection{Hidroxil propil metil celulose ftalato (HPMCP)}

HPMCP é um pó granular ou em flocos de fluxo livre, branco a ligeiramente esbranquiçado, com um odor levemente ácido e um sabor quase imperceptível. É um derivado da hidroxipropilmetilcelulose produzida pela transesterificação da hidroxipropilmetilcelulose com ácido ftálico (ARAI, GALLERANI, 20II).

Este polímero foi introduzido pela primeira vez em 1971 pela Shin-Etsu Chemical Company, Tóquio, Japão como um derivado de celulose para revestimento entérico. HPMCP foi admitido nas farmacopéias européia e japonesa e incluído no USP / NF sob o nome de ftalato de hipromelose. Dependendo do grau de substituição do ftalil, o HPMCP é solúvel em meio aquoso em uma faixa de $\mathrm{pH}$ de 5,0 - 5,5 (MIRANDA, 2015). HPMCP é caracteristicamente insolúvel em fluidos gástricos, mas incha e rapidamente solúvel na parte superior do intestino. Pode ser plastificado com dietilftalato,

monoglicerídeo acetilado ou triacetina. Mecanicamente, o HPMCP é um polímero mais flexível e em uma base de peso não requer tanto plastificante quanto CAP ou CAT (TESTA, 2013).

insolúvel em diclorometano, metanol, isopropanol. acetato de etilo e etanol, mas demonstra a solubilidade desejada em acetona, tetra-hidrofurano, misturas de diclorometano e metanol, misturas de diclorometano e etanol e misturas de acetona e metanol. A insolubilidade desse polímero em sistema de solvente único torna um desafio conduzir estudos simples de compatibilidade de drogas e aplicações de secagem por pulverização (ARAI, GALLERANI, 20II). No entanto, as misturas de solventes podem ser efetivamente preparadas para secagem por pulverização comercial usando a otimização de secagem por pulverização adequada. Esses polímeros permanecem química e fisicamente estáveis em temperatura ambiente por vários anos, mas são suscetíveis à hidrólise em condições de temperatura e umidade elevadas (TESTA, 2013).

I.I.4. Succinato de acetato de hidroxil propil metil celulose (HPMCAS)

HPMCAS também conhecido como succinato de acetato de hipromelose é um pó branco a esbranquiçado ou grânulos derivados de HPMC pela esterificação de grupos hidroxila livres na estrutura do polímero com anidrido acético e anidrido succínico. Está 
disponível comercialmente em três graus ( $L, M$ e $H$ ), que correspondem a perfis de liberação dependentes do $\mathrm{pH}$ de baixo $\mathrm{pH}(5,0)$, médio $(5,5)$ e alto $(6,5) \mathrm{Ph}$ (ARAI, GALLERANI, 20II).

Figura 3: ESTRUTURA QUÍMICA ANIDRIDO ACÉTICO

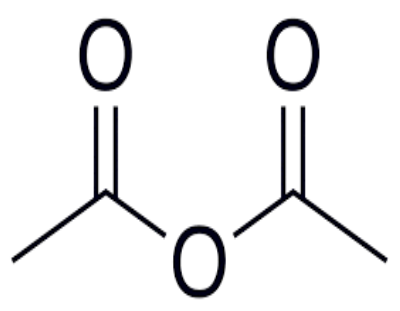

Figura 4: ESTRUTURA QUÍMICA ANIDRIDO SUCCÍNICO

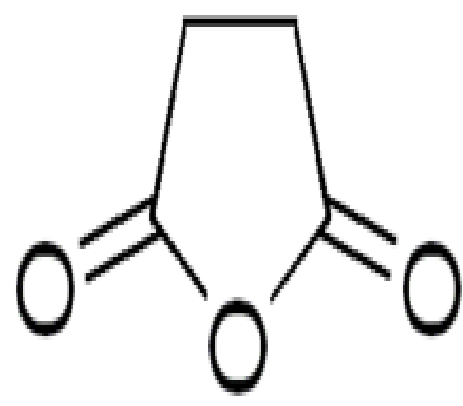

HPMCAS é insolúvel em meio ácido, mas solúvel em $\mathrm{pH}$ neutro de acordo com a ionização de grupos carboxila livres na estrutura do polímero. Foi demonstrado que é mais estável quimicamente do que CAP e HPMCP, conforme indicado por uma redução substancial na evolução de ácido livre quando armazenado a $60^{\circ} \mathrm{C}$ e $100 \%$ de umidade relativa (UR) (TESTA, 2013). HPMCAS tem temperatura de transição vítrea variando entre $120^{\circ} \mathrm{C}-135^{\circ} \mathrm{C}$ de acordo com o grau de polímero. Uma vez que HPMCAS é um polímero relativamente rígido, a plastificação é utilizada para melhorar a flexibilidade do filme e reduzir rachaduras, bem como para promover a formação de filme a partir de dispersões aquosas de HPMCAS (ARAI, GALLERANI, 20II). 
I.I.5. Acetato ftalato de polivinila (PVAP)

O ftalato de acetato de polivinila é um pó branco a esbranquiçado de fluxo livre com um leve odor de ácido acético. É um produto da reação de anidrido ftálico, acetato de sódio e um álcool polivinílico parcialmente hidrolisado. $\mathrm{O}$ início da dissolução aquosa de PVAP começa a um $\mathrm{pH}$ de cerca de 5,o, permitindo a liberação entérica, bem como o potencial de liberação direcionada do fármaco para o intestino delgado proximal. Embora estruturalmente semelhante ao CAP (contendo o ácido ftálico dicarboxílico em uma forma parcialmente esterificada) (TESTA, 2013).

Figura 5: ESTRUTURA QUÍMICA ÁLCOOL POLIVINÍLICO

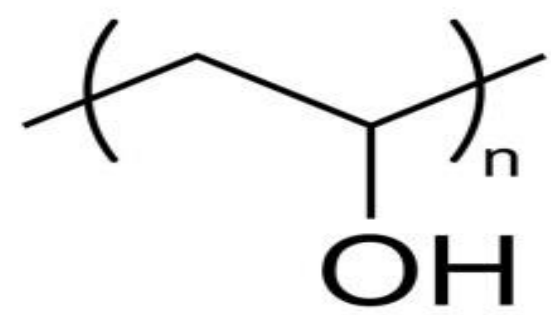

O PVAP é relativamente mais estável à hidrólise do que o CAP devido à sua menor permeabilidade à umidade. É compatível com vários dos plastificantes mais comuns; nomeadamente triacetato de glicerilo, TEC, trietilcitrato de acetilo, DEP e PEG 40o. PVAP (Sureteric ${ }^{\circledR}$ ) está disponível comercialmente na Colorcon como um sistema de revestimento pré-formulado completo que consiste em uma mistura de pó de PVAP, plastificantes e outros ingredientes funcionais destinados à reconstituição em água para rápida produção de dispersão de revestimento (TESTA, 2013).

I.r.6. Copolímeros de ácido metacrílico

Este grupo de polímeros é amplamente utilizado para aplicações de revestimento entérico, pois contém grupos de ácido carboxílico livres que são ionizados sempre que o $\mathrm{pH}$ do ambiente excede 5,5. Eles são produzidos por um processo de polimerização em emulsão e foram introduzidos pela primeira vez para aplicações de revestimento entérico por Lehmann e Dreher em meados da década de 1960. Hoje, esses polímeros entéricos são 
comercializados principalmente pela Evonik (anteriormente conhecida como Röhm $\mathrm{GmbH}$ ) sob a marca Eudragit (ARAI, GALLERANI, 20II).

Figura 6: ESTRUTURA QUIIMICA ÁCIDO METACRÍLICO

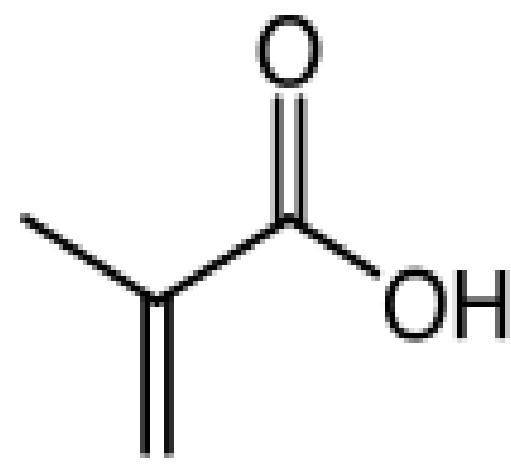

I.2. Vários tipos diferentes de polímeros Eudragit com capacidade de liberação entérica estão disponíveis comercialmente em uma ampla gama de diferentes formas físicas (dispersão aquosa, solução orgânica, grânulos e pós). $\mathrm{O} \mathrm{pH}$ no qual esses polímeros se dissolvem depende do teor de ácido carboxílico no copolímero. Copolímeros de metilmetacrilato de ácido metacrílico (Eudragit L e S) e copolímero de etil acrilato de ácido metacrílico (Eudragit $\mathrm{L}_{30} \mathrm{D}$ ) são a escolha preferida de polímeros de revestimento para formulações entéricas. Eles permitem o direcionamento de áreas específicas do intestino (TESTA, 2013).

\section{I.3 FATORES QUE PODEM INFLUENCIAR O COMPORTAMENTO DE POLÍMEROS DE REVESTIMENTO ENTÉRICO}

A funcionalidade dos polímeros de revestimento entérico pode ser muito afetada por fatores, tais como

i. A natureza da substância medicamentosa contida na forma de dosagem; isso é especialmente verdadeiro quando a substância medicamentosa é de natureza iônica;

ii. A quantidade de revestimento aplicado; a aplicação de revestimento excessivo pode retardar substancialmente a liberação do fármaco da forma de dosagem, enquanto o revestimento insuficiente pode resultar em resistência gástrica ineficaz;

iii. A presença de imperfeições no revestimento (por exemplo, rachaduras, “marcas de arranhões", etc.) pode destruir a integridade do revestimento;

iv. $\mathrm{O} \mathrm{pH}$ de dissolução e a taxa de dissolução do polímero usado no revestimento; 
v. A influência das condições de teste in vitro usadas (como $\mathrm{pH}$ e força iônica da solução de teste; bem como a taxa de agitação usada no teste);

vi. A presença ou ausência de plastificantes;

vii. Fatores fisiológicos gastrointestinais: $\mathrm{O} \mathrm{pH}$ do estômago e do conteúdo intestinal; Esvaziamento gástrico e Atividade enzimática do trato gastrointestinal (TESTA, 2013).

\section{CONCLUSÃO}

A maioria das cápsulas farmacêuticas é feita de gelatina ou de amido sendo obtido das raizes da mandioca e tratada quimicamente para obter hidroxipropil amido de tapioca. Esses materiais se dissolvem nos sucos gástricos de baixo $\mathrm{pH}$ no estômago. Alguns medicamentos, entretanto, não são eficazes se forem liberados no estômago e precisam passar pelos intestinos, onde podem ser absorvidos em $\mathrm{pH}$ mais alto. As cápsulas com revestimento entérico foram concebidas para passar através do estômago sem ser afetado e para se dissolver no trato intestinal.

As cápsulas com revestimento entérico não se dissolvem na boca e, portanto, são perfeitas para formulações que não precisam ser ativadas por exposição a ácidos. $\mathrm{O}$ revestimento evita que a cápsula se dissolva na boca e nas áreas do esôfago. Muitos medicamentos podem causar irritação no estômago se forem liberados lá; com cápsulas com revestimento entérico, a formulação passa pelo estômago sem irritar o revestimento do estômago.

O revestimento entérico também evita que qualquer enzima presente na cápsula se dissolva no ambiente ácido da área do estômago. Esta cápsula com revestimento entérico normalmente leva uma ou duas horas para se dissolver, tornando-a ideal para medicamentos sensíveis ao tempo.

\section{REFERÊNCIAS BIBLIOGRÁFICAS}

ARAI, A. E; GALLERANI, S. M. C. USO CRÔNICO DE FÁRMACOS INIBIDORES
DA BOMBA DE PRÓtONS: EFICÁCIA CLÍNICA E EFEITOS ADVERSOS. Monografia apresentada ao curso de Especialização em Farmacologia. Londrina, 2oIr. 
DEPONTI, V. B. Cápsulas de liberação modificada: é possível produzir em Farmácia? Trabalho de Conclusão da Disciplina de Estágio Curricular em Farmácia. Porto Alegre, junho de 2012.

MENDONÇA, C. F. do V. DESENVOLVIMENTO E AVALIAÇÃO DE REVESTIMENTO APLICADO A CÁPSULAS DE GELATINA DURA GASTRORESISTENTES EM ESCALA MAGISTRAL. Dissertação apresentada ao Programa de Pós-graduação em Ciências Farmacêuticas da Universidade de Sorocaba, como exigência parcial para obtenção do título de Mestre. Sorocaba/SP 2010.

MIRANDA, B. Q. S. ESTUDO DE UTILIZAÇÃO DE INIBIDORES DA BOMBA DE PRÓTONS EM FARMÁCIAS COMUNITÁRIAS PRIVADAS DO SUBMÉDIO DO VALE DO SÃO FRANCISCO. Trabalho apresentado a Universidade Federal do Vale do São Francisco - UNIVASF, Campus Petrolina, como requisito da obtenção do título de Bacharel em Farmácia. PETROLINA, 2015.

TESTA, C. G. Avaliação comparativa do perfil de dissolução in vitro de microgrânulos gastrorresistentes de omeprazol de diferentes fabricantes para desenvolvimento de medicamento similar. Monografia apresentada ao Curso de Pósgraduação Lato Sensu como requisito para obtenção do título de Especialista em Tecnologias Industriais Farmacêuticas. Rio de Janeiro, 2013. 